ISSN 1980-5098

\title{
CRESCIMENTO E QUALIDADE DE MUDAS DE Cassia grandis Linnaeus f. EM RESPOSTA À ADUBAÇÃO FOSFATADA E CALAGEM
}

\author{
GROWTH AND QUALITY OF Cassia grandis Linnaeus f. SEEDLINGS IN RESPONSE TO \\ PHOSPHATE FERTILIZATION AND LIMING
}

\author{
Eliane Cristina Sampaio de Freitas ${ }^{1}$ Haroldo Nogueira de Paiva ${ }^{2}$ Helio Garcia Leite ${ }^{2}$ \\ Silvio Nolasco de Oliveira Neto ${ }^{2}$
}

\begin{abstract}
RESUMO
O objetivo deste estudo foi avaliar o crescimento e a qualidade de mudas de Cassia grandis Linnaeus $\mathrm{f}$. (cássia-rosa) em função da adubação fosfatada e da calagem. Os tratamentos foram representados por um fatorial de seis níveis de fósforo $\left(0,120,240,360,480\right.$ e $\left.600 \mathrm{mg} \mathrm{dm}^{-3}\right)$ por cinco níveis de saturação por bases do substrato (3,5 (original), 25, 40, 55, 70\%), sendo dispostos no delineamento em blocos ao acaso, com quatro repetições. Aos 65 dias após a repicagem, foram medidos a altura da parte aérea $(\mathrm{H})$, o diâmetro do coleto (DC), a massa de matéria seca da parte aérea (MSPA), massa de matéria seca de raízes (MSRA) e massa de matéria seca total (MST), e calculada a relação MSPA/MSRA e o índice de qualidade de Dickson (IQD). A adubação fosfatada teve influência significativa em todas variáveis estudadas. A dose de fósforo adequada para a produção de mudas de qualidade de Cassia grandis nas condições estudadas, na saturação por bases de $25 \%$, é de $600 \mathrm{mg} \mathrm{dm}^{-3}$.
\end{abstract}

Palavras-chave: produção de mudas; nutrição de plantas; fósforo.

\begin{abstract}
This study aimed at evaluating the growth and seedling quality of Cassia grandis Linnaeus $\mathrm{f}$. in response to liming and phosphate fertilization. The treatments were arranged in a factorial design of 6 phosphorus levels $\left(0,120,240,360,480\right.$ and $\left.600 \mathrm{mg} \mathrm{dm}^{-3}\right)$ by 5 base saturation levels (3,5 (original), 25, 40, 55, 70 $\%$, in randomized blocks with four replicates. The height of the aerial part (H), collar diameter (DC), dry matter of shoot (MSPA), dry matter of root (MSRA), and total dry matter (MST) were determined at 65 days after the transplanting. The relation between dry matter of shoot and dry matter of root (MSPA/ MSRA) and Dickson quality index (IQD) were calculated at this stage. There were significant effects from the phosphate fertilization to all studied variables. Based on this study, the recommended $\mathrm{P}$ dose for quality seedling production of Cassia grandis is $600 \mathrm{mg} \mathrm{dm}^{-3}$, for $25 \%$ of substratum's base saturation.
\end{abstract}

Keywords: seedling production; plant nutrition; phosphorus.

\section{INTRODUÇÃO}

A expansão agrícola e a alta demanda por produtos madeireiros aumentaram a exploração dos remanescentes florestais nativos, resultando em altas taxas de desmatamento, que culminaram na degradação de extensas áreas no Brasil devido ao seu abandono após a perda da capacidade produtiva ou manejo incorreto. A revegetação dessas áreas é fundamental para melhorar as características físicas e químicas do solo, fornecendo, por meio da cobertura vegetal, a proteção necessária para diminuir a perda de solo por erosão (SILVA et al., 2011).

1 Engenheira Florestal, MSc., Doutoranda em Ciência Florestal, Departamento de Engenharia Florestal, Universidade Federal de Viçosa, Av. P. H. Rolfs, s/n, Campus Universitário, CEP 36570-000, Viçosa (MG), Brasil. elianesampaiofreitas@hotmail.com

2 Engenheiro Florestal, Dr., Professor do Departamento de Engenharia Florestal, Universidade Federal de Viçosa, Av. P. H. Rolfs, s/n, Campus Universitário, CEP 36570-000, Viçosa (MG), Brasil. hnpaiva@ufv.br/ hgleite@ufv.br/snolasco@ufv.br 
Os projetos de revegetação, que visam à recuperação de áreas degradadas e proteção de mananciais hídricos, recomendam o plantio de espécies nativas, tendo em vista reestabelecer as condições ecológicas (FURTINI NETO et al., 1999). A utilização de espécies arbóreas nativas, adaptadas às condições de estresse, pode reverter os processos de degradação por meio da estabilização do solo, pelo desenvolvimento do sistema radicular e aumento da matéria orgânica do solo através da deposição de serapilheira (PARROTTA, 1992).

A Cassia grandis, popularmente conhecida como cássia-rosa, é uma espécie pioneira a secundária inicial (CARVALHO, 1994). Pertencente à família Fabaceae e subfamília Caesalpinioideae, com ocorrência no Amazonas, Bahia, Maranhão, Mato Grosso, Mato Grosso do Sul, Pará, Paraíba, Pernambuco, Rio de Janeiro, Roraima, Sergipe e Tocantins (CARVALHO, 2006), essa espécie apresenta melhor crescimento em solos com elevada fertilidade, em plantios experimentais (CARVALHO, 1994).

Além de ser muito utilizada para o paisagismo, uma vez que é extremamente ornamental (LORENZI, 2002), para a consorciação em sistemas agroflorestais, devido aos frutos adocicados que são apreciados pelo gado, e utilização de sua madeira na construção civil ou para produção de carvão (CARVALHO, 1994), a cássia-rosa é utilizada para a recuperação de matas ciliares, em locais com inundações periódicas de rápida duração e com curto período de encharcamento.

Apesar de onerosa, a intervenção por meio do plantio de mudas para a recuperação de áreas degradadas, em muitos casos, pode ser mais eficaz em relação ao tempo de estabelecimento. A produção de mudas é uma das fases mais importantes para os projetos de florestamento, seja para fins comerciais ou ambientais. Mudas de espécies arbóreas de boa qualidade, com nutrição e substratos adequados, são fundamentais para garantir a adaptação e crescimento após o plantio (GONÇALVES et al., 2008), evitando replantios e gastos adicionais (GOMES et al., 2002).

Entre os substratos utilizados para a produção de mudas de espécies arbóreas nativas, destacase a terra de subsolo, que ainda é muito utilizada pelos viveiros. Todavia, a maioria dos solos brasileiros apresenta elevada acidez e baixa disponibilidade de nutrientes em condições naturais, sendo o principal deles o fósforo. Nesse sentido, são necessárias práticas que corrijam essas propriedades do solo, possibilitando o desenvolvimento das espécies cultivadas. A calagem é uma prática que objetiva a correção da acidez do solo, e também o aumento da disponibilidade da maioria dos nutrientes para as plantas (ALVAREZ; RIBEIRO, 1999), como o fósforo, uma vez que eleva o $\mathrm{pH}$, e, consequentemente, a densidade de cargas negativas, acarretando maior repulsão entre o fósforo e a superfície adsorvente e redução da capacidade máxima de adsorção do solo (NOVAIS et al., 2007).

Segundo Alvarez e Ribeiro (1999), os critérios de recomendação da calagem são variáveis de acordo com o objetivo dessa prática. Entre os métodos utilizados para determinar a necessidade da calagem, quantidade de corretivo a ser aplicada, esses autores citam o Método da neutralização da acidez trocável e da elevação dos teores de Ca e de Mg trocáveis e o Método da saturação por bases. No primeiro, são consideradas as características do solo e as exigências das culturas, objetivando reduzir a acidez do solo, levando em conta a máxima saturação por $\mathrm{Al}^{3+}$ tolerada pela cultura e a capacidade tampão do solo, e elevar a disponibilidade de $\mathrm{Ca}$ e $\mathrm{Mg}$ de acordo com as exigências das culturas (ALVAREZ; RIBEIRO, 1999). Entretanto, as informações a respeito da máxima saturação por $\mathrm{Al}^{3+}$ tolerada pelas culturas, assim como o requerimento de $\mathrm{Ca}$ e $\mathrm{Mg}$, são desconhecidas para a maioria das espécies arbóreas nativas. O segundo método tem como intuito fazer a calagem para atingir desejado valor de saturação por bases, corrigindo a acidez do solo até definido $\mathrm{pH}$, considerado adequado para determinada cultura (ALVAREZ; RIBEIRO, 1999). Nesse método é considerada a relação existente entre o $\mathrm{pH}$ e a saturação por bases, sendo que o pH do solo aumenta a medida que a saturação por bases aumenta (VAN RAIJ; SANCCHETTO, 1968).

O fósforo é fundamental nos processos de armazenamento e transferência de energia (MARSCHNER, 1995), e sua deficiência é um dos fatores que mais limita o crescimento das espécies arbóreas, sendo responsável pelo inadequado desenvolvimento das plantas em solos ácidos de regiões tropicais (FURTINI NETO et al., 2005). A deficiência de fósforo nos substratos provoca crescimento irregular, tanto na parte aérea quanto no sistema radicular, prejudicando a qualidade das mudas (GOMES; PAIVA, 2012). Nesse sentido, adubações fosfatadas são necessárias para atender à demanda das plantas, que é variável de acordo com a espécie. Contudo, o conhecimento sobre as exigências nutricionais das espécies arbóreas nativas é escasso, justificando a realização de estudos que visam obter informações para a produção de mudas 
com melhor qualidade (CRUZ et al., 2004), e otimizar o uso dos fertilizantes, atentando para os fatores econômicos e ambientais.

Diante a importância das espécies arbóreas nativas para diversos projetos de florestamento e escassez de informações sobre suas exigências nutricionais, o presente estudo teve como objetivo avaliar a influência da adubação fosfatada e da calagem sobre o crescimento e a qualidade de mudas de Cassia grandis.

\section{MATERIAIS E MÉTODOS}

O estudo foi conduzido entre outubro de 2012 e janeiro de 2013, em Viçosa - MG, localizada a $20^{\circ} 45^{\prime}$ de latitude Sul e $46^{\circ} 51^{\prime}$ de longitude Oeste, a 689 metros de altitude, no Viveiro de Pesquisa do Departamento de Engenharia Florestal da Universidade Federal de Viçosa. Segundo a classificação de Köppen, o clima na região de Viçosa é do tipo Cwb, mesotérmico com verões quentes e chuvosos, e invernos frios e secos. A temperatura e a precipitação pluviométrica média anual são de $19,4^{\circ} \mathrm{C}$ e $1.221 \mathrm{~mm}$, respectivamente (INSTITUTO NACIONAL DE METEOROLOGIA, 1992).

O substrato utilizado foi um Latossolo Vermelho-Amarelo Distrófico (EMBRAPA, 2013), textura argilosa, retirado na camada de $20-50 \mathrm{~cm}$ de profundidade. Posteriormente, o solo foi peneirado (malha de $4 \mathrm{~mm}$ de diâmetro), ocasião em que foi coletada amostra para caracterização física (argila $=570 \mathrm{~g} \mathrm{~kg}^{-1}$, silte $=110 \mathrm{~g} \mathrm{~kg}^{-1}$, areia grossa $=190 \mathrm{~g} \mathrm{~kg}^{-1}$ e areia fina $=130 \mathrm{~g} \mathrm{~kg}^{-1}$ ) e química, segundo Donagema (2011) (Tabela 1).

Os tratamentos foram representados por um fatorial de seis níveis de $\mathrm{P}(0,120,240,360,480 \mathrm{e}$ $600 \mathrm{mg} \mathrm{dm}^{-3}$ ), por cinco níveis de saturação por bases (3,5 (original), 25, 40, 55 e 70\%), sendo dispostos no delineamento em blocos ao acaso, com quatro repetições. A parcela experimental foi constituída por um vaso de polietileno rígido, contendo $1,8 \mathrm{dm}^{3}$ de solo.

O solo recebeu cinco níveis de calagem, de acordo com o método da saturação por bases, ou seja, $3,5 \%$ (saturação por bases em condições naturais), e elevação da saturação por bases para 25, 40, 55 e 70\%, utilizando a seguinte fórmula para cálculo (ALVAREZ; RIBEIRO, 1999):

$$
N C=\frac{\left[(V 2-V 1) \times C T C_{p H 7,0}\right]}{100}
$$

Em que: $\mathrm{NC}=$ necessidade de calagem $\left(\right.$ toneladas hectare $\left.{ }^{-1}\right) ; \mathrm{V} 2=$ porcentagem de saturação por bases desejada; $\mathrm{V} 1$ = porcentagem de saturação por bases do solo, conforme análise; $\mathrm{CTC}_{\mathrm{pH} 7,0}=$ Capacidade de troca catiônica.

O corretivo consistiu em uma mistura de $\mathrm{CaCO}_{3}$ e $\mathrm{MgCO}_{3}$, na relação estequiométrica de 4:1. Após a aplicação do corretivo, o solo foi incubado durante 30 dias, com o teor de umidade mantido próximo à capacidade de campo, incluindo a porção que não recebeu calcário, sendo então destinado ao preenchimento dos vasos.

A fonte de $\mathrm{P}$ utilizada foi o fosfato de sódio monobásico-mono-hidratado $\left(\mathrm{NaH}_{2} \mathrm{PO}_{4} \cdot \mathrm{H}_{2} \mathrm{O}\right)$ aplicado por solução antes da repicagem. A adubação com macro e micronutrientes foi baseada em recomendações para outras espécies perenes, uma vez que não existem recomendações para a espécie estudada. A adubação

TABELA 1: Características químicas da amostra do solo utilizado para a produção de mudas de cássia-rosa (Cassia grandis Linnaeus f.).

TABLE 1: Chemical analysis of the sample of soil used in the seedling production of Cassia grandis Linnaeus $\mathrm{f}$.

\begin{tabular}{|c|c|c|c|c|c|c|c|c|c|c|c|c|}
\hline $\mathrm{pH}$ & $P$ & K & $\mathrm{Al}^{3+}$ & $\mathrm{Ca}^{2+}$ & $\mathrm{Mg}^{2+}$ & $\mathrm{H}+\mathrm{Al}$ & SB & $\mathrm{CTC}_{\mathrm{ef}}$ & $\mathrm{CTC}_{\mathrm{pH} 7,0}$ & $\mathrm{~V}$ & $\mathrm{Al}$ & MO \\
\hline $\mathrm{H}_{2} \mathrm{O}$ & \multicolumn{2}{|c|}{$--\mathrm{mg} \mathrm{dm}^{-3}--$} & --- & 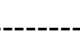 & -.-- & $--\mathrm{cm}$ & $\mathrm{dm}^{-3}$ & 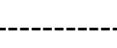 & -------- & ------- & \%)----.- & dag $\mathrm{kg}^{-1}$ \\
\hline 4,7 & 0,70 & 6,00 & 0,92 & 0,11 & 0,01 & 3,93 & 0,14 & 1,06 & 4,04 & 3,5 & 86,80 & 1,66 \\
\hline
\end{tabular}


básica foi aplicada, 15 dias após a repicagem, por solução e consistiu de $100 \mathrm{mg} \mathrm{dm}^{-3} \mathrm{de} \mathrm{N}, 100 \mathrm{mg} \mathrm{dm}^{-3}$ de $\mathrm{K}$ e $40 \mathrm{mg} \mathrm{dm}^{-3}$ de $\mathrm{S}$, pelo emprego de $\mathrm{NH}_{4} \mathrm{NO}_{3}, \mathrm{KCl}_{\text {e }} \mathrm{K}_{2} \mathrm{SO}_{4}$, respectivamente, conforme sugerido por Passos (1994). Após 30 dias, foi realizada uma adubação de cobertura de $50 \mathrm{mg} \mathrm{dm}^{-3} \mathrm{de} \mathrm{N}$. Juntamente à primeira aplicação foi realizada uma adubação com solução de micronutrientes nas seguintes doses e fontes: $0,81 \mathrm{mg} \mathrm{dm}^{-3} \mathrm{de} \mathrm{B}\left(\mathrm{H}_{3} \mathrm{BO}_{3}\right), 1,33 \mathrm{mg} \mathrm{dm}{ }^{-3} \mathrm{de} \mathrm{Cu}\left(\mathrm{CuSO}_{4} \cdot 5 \mathrm{H}_{2} \mathrm{O}\right), 3,66 \mathrm{mg} \mathrm{dm}{ }^{-3} \mathrm{de} \mathrm{Mn}\left(\mathrm{MnCl}_{2} \cdot \mathrm{H}_{2} \mathrm{O}\right)$, $4,00 \mathrm{mg} \mathrm{dm}^{-3}$ de $\mathrm{Zn}\left(\mathrm{ZnSO}_{4} \cdot 7 \mathrm{H}_{2} \mathrm{O}\right)$, e $0,15 \mathrm{mg} \mathrm{dm}{ }^{-3} \mathrm{de} \mathrm{Mo}\left(\left(\mathrm{NH}_{4}\right)_{6} \mathrm{Mo}_{7} \mathrm{O}_{24} \cdot 4 \mathrm{H}_{2} \mathrm{O}\right)$, de acordo com Alvarez et al. (2006).

As sementes de cássia-rosa foram tratadas com ácido sulfúrico por 40 minutos para a quebra de dormência, e semeadas em canteiro de areia. Após 24 dias, quando apresentavam dois pares de folhas, duas mudas foram repicadas para cada vaso e mantidas por 15 dias em casa de sombra (sombrite $50 \%$ ). Posteriormente, as mudas foram colocadas em casa de vegetação e raleadas, deixando apenas uma muda por vaso. Durante o período experimental, a umidade do solo foi monitorada diariamente, sendo feita a irrigação conforme a necessidade.

Aos 65 dias após a repicagem, foram feitas medições da altura e diâmetro do coleto de todas as plantas utilizando régua graduada em centímetros e paquímetro digital com precisão de $0,01 \mathrm{~mm}$, respectivamente. As plantas foram separadas em raiz e parte aérea, lavadas e secas em estufa com circulação forçada de ar, a uma temperatura próxima a $70^{\circ} \mathrm{C}$, até peso constante. Em seguida, as partes constituintes das plantas foram pesadas em balança analítica com precisão de $0,01 \mathrm{~g}$.

Através dos dados de altura $(\mathrm{H})$, diâmetro do coleto $(\mathrm{DC})$, massa de matéria seca da parte aérea (MSPA), raiz (MSRA) e total (MST), foram calculadas as relações de qualidade de mudas: MSPA/MSRA e o Índice de Qualidade de Dickson (IQD), de acordo com a fórmula:

$$
I Q D=M S T \div[(H \div D C)+(M S P A \div M S R A)]
$$

Os dados foram analisados por meio da análise de variância e de regressão $(\mathrm{p}<0,05)$. Foram ajustadas equações de regressão em função das doses de P aplicadas, dentro de cada nível de calagem. Os modelos foram escolhidos baseados na significância dos coeficientes da regressão e no coeficiente de determinação $\left(\mathrm{R}^{2}\right)$. Também foi calculado o coeficiente de correlação de Pearson para avaliar a correlação entre as variáveis estudadas.

\section{RESULTADOS E DISCUSSÃO}

A adubação fosfatada teve influência significativa em todas as características avaliadas e suas relações (Tabela 2). Apenas o IQD apresentou interação significativa entre a adubação fosfatada e a calagem. A calagem não apresentou efeito significativo para as características avaliadas, exceto para a relação MSPA/ MSRA. A falta de resposta à calagem pode ser explicada pela adaptação da espécie a solos ácidos, e que os teores de $\mathrm{Ca}$ e $\mathrm{Mg}$ contidos no solo atendem às exigências da espécie na fase inicial de crescimento. A resposta à calagem pode ou não ocorrer, dependendo das características de cada espécie que se pretenda cultivar, principalmente no que se refere à tolerância a acidez (VALE et al., 1996).

A ausência de resposta significativa para a calagem também foi observada para a Anadenanthera colubrina (GOMES et al., 2004); Acacia holoserica e Acacia auriculiformis (BALIEIRO et al., 2001); Tabebuia impetiginosa (CRUZ et al., 2004); Astronium fraxinifolium, Guazuma ulmifolia, Anadenanthera macrocarpa e Inga edulis (SILVA; PEREIRO; RODRIGUES, 2011). Algumas espécies desenvolvem mecanismos para se adaptar a solos ácidos, como por exemplo, espécies tolerantes à toxidez por $\mathrm{Al}$ que reduzem sua absorção aumentando o pH da rizosfera ou excluindo-o do tecido (VALE et al., 1996).

A resposta à calagem também pode ser influenciada pelo tipo de substrato utilizado para a produção de mudas. Para a produção de mudas de angico-vermelho (Anadenanthera macrocarpa), fedegoso (Senna macranthera) e bico-de-pato (Machaerium nictitans), em Argissolo, Bernardino et al. (2005), Souza et al. (2010) e Souza et al. (2008), respectivamente, não observaram efeito significativo da elevação da saturação por bases sobre as características morfológicas e suas relações. Por outro lado, esses autores observaram resposta significativa dessas espécies quando utilizaram como substrato Latossolos distrófico e álico. 
TABELA 2: Resumo da análise de variância das características morfológicas e suas relações para mudas de Cassia grandis, aos 65 dias após a repicagem, em resposta às doses de fósforo $(\mathrm{P})$ e saturação por bases do substrato $(\mathrm{V})$.

TABLE 2: Summary of the variance analysis of the morphologic characteristics and relations studied in the production of Cassia grandis seedlings in response to substratum's base saturation (V) and phosphate fertilization $(\mathrm{P})$. The morphologic characteristics were evaluated at 65 days after transplanting.

\begin{tabular}{lcccccccc}
\hline \multirow{2}{*}{ Fonte de variação } & \multirow{2}{*}{$\mathrm{GL}$} & \multicolumn{7}{c}{ Quadrado médio } \\
\cline { 3 - 9 } & & $\mathrm{H}$ & $\mathrm{DC}$ & MSPA & MSRA & MST & MSPA/MSRA & IQD \\
\hline Bloco & 3 & 1,64 & 0,56 & 0,08 & 0,01 & 0,06 & $0,33^{*}$ & 0,00 \\
$\mathrm{~V}$ & 4 & 4,62 & 0,27 & 0,37 & 0,16 & 0,98 & $0,22^{*}$ & 0,02 \\
$\mathrm{P}$ & 5 & $298,13^{* *}$ & $5,16^{* *}$ & $7,67^{* *}$ & $1,45^{* *}$ & $15,79^{* *}$ & $0,65^{* *}$ & $0,21^{* *}$ \\
PxV & 20 & 17,91 & 0,43 & 0,52 & 0,13 & 1,15 & 0,08 & $0,02^{*}$ \\
Resíduo & 87 & 10,64 & 0,27 & 0,36 & 0,1 & 0,79 & 0,09 & 0,01 \\
\hline \multicolumn{1}{c}{ CV \% } & & 14,17 & 13,46 & 32,74 & 34,18 & 32,56 & 14,52 & 31,13 \\
\hline
\end{tabular}

Em que: ${ }^{* *} \mathrm{e}^{*}$ significativo a $1 \%$ e $5 \%$ de probabilidade, respectivamente, pelo teste $\mathrm{F}$. $\mathrm{H}$ - altura da parte aérea; $\mathrm{DC}$ - diâmetro do coleto; MSPA - massa de matéria seca da parte aérea; MSRA - massa de matéria seca da raiz; MST massa de matéria seca total; MSPA/MSRA - relação entre massa de matéria seca da parte aérea e massa de matéria seca da raiz; IQD - índice de qualidade Dickson.

Respostas significativas para a calagem, contrárias às obtidas nesse estudo, favorecendo o crescimento em altura, diâmetro e produção de matéria seca de mudas, foram encontradas para a cássiaverrugosa (Senna multijuga), ipê-mirim (Stenolobium stans), angico-do-cerrado (Anadenanthera falcata) e cedro (Cedrela fissilis) (FURTINI NETO et al., 1999). Melhores mudas de nim-indiano (Azadirachta indica) foram produzidas quando a saturação por bases do substrato estava entre 30 e $40 \%$ (CALDEIRA JÚNIOR et al., 2007), e 50\% para angico-vermelho (Anadenanthera macrocarpa) (BERNARDINO et al., 2005) e ipê-roxo (Tabebuia impetiginosa) (CRUZ et al., 2004). Ressalta-se que as respostas das espécies devem ser comparadas com mudas produzidas em substratos com características químicas e físicas semelhantes.

Algumas características apresentaram respostas quadráticas em função da adubação fosfatada (Figura 1 e Tabela 3), sendo possível obter o ponto de máximo crescimento em resposta às doses de fósforo estudadas.

Entre as características morfológicas que determinam a qualidade das mudas, a altura da parte aérea é muito utilizada em viveiros, pois, além de sua medição ser fácil e não destrutiva (GOMES et al., 2002), fornece uma excelente estimativa da predição do crescimento inicial no campo (CAIONE; LANGE; SCHONINGER, 2012). A maior altura $(27,37 \mathrm{~cm})$ foi obtida na dose de $319,99 \mathrm{mg} \mathrm{dm}^{-3}$ de $\mathrm{P}$ na saturação por bases de $55 \%$. Valores próximos de altura foram obtidos com maiores doses, 413 e $525 \mathrm{mg} \mathrm{dm}^{-3} \mathrm{de}^{-}$ $\mathrm{P}$, nas saturações por bases de $25 \%$ e $70 \%$, respectivamente. O valor máximo encontrado para a altura se enquadra no estabelecido por Gonçalves et al. (2005) para mudas de boa qualidade, que possuem entre 20 a $35 \mathrm{~cm}$ de altura.

Na saturação por bases de $55 \%$, a dose que possibilitou o maior diâmetro do coleto $(4,41 \mathrm{~mm})$ foi a de $323,93 \mathrm{mg} \mathrm{dm}^{-3}$ de P. Na saturação por bases natural do solo, a resposta foi linear positiva e o diâmetro obtido com a maior dose de $\mathrm{P}$ foi de $4,50 \mathrm{~mm}$. A sobrevivência e o crescimento inicial das mudas no campo são relacionadas diretamente com o diâmetro do coleto (SOUZA et al., 2006) e altura da parte aérea (GOMES; PAIVA, 2012). Santin et al. (2008) também obtiveram resposta quadrática para as características avaliadas em mudas de erva-mate (Ilex paraguariensis), com os valores máximos obtidos nas doses de 440 e $533 \mathrm{mg} \mathrm{dm}^{-3}$ de P para diâmetro do coleto e altura, respectivamente.

Os maiores valores de massa de matéria seca da parte aérea $(2,82 \mathrm{~g})$, de raízes $(1,33 \mathrm{~g})$ e total $(3,51$ g), na saturação por bases de $25 \%$, foram obtidos com doses no intervalo de 490 a $600 \mathrm{mg} \mathrm{dm}^{-3} \mathrm{de}$ P. A massa de matéria seca é a característica que melhor reflete a produção (GONÇALVES et al., 2008) e tem 
(a)

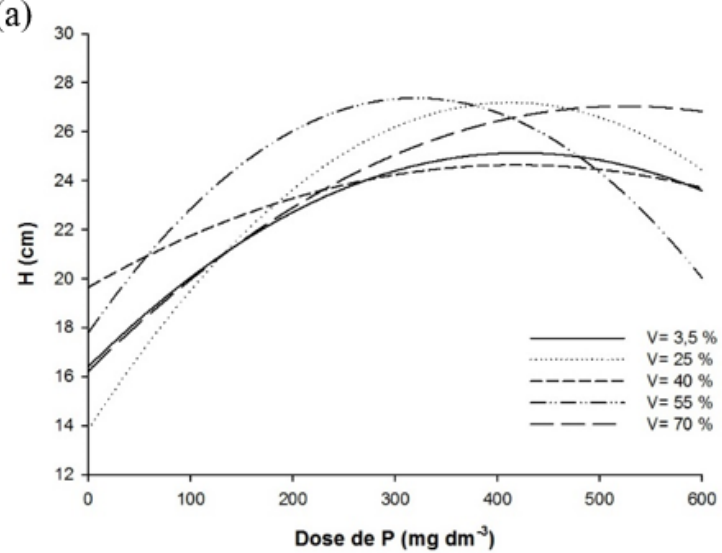

(c)

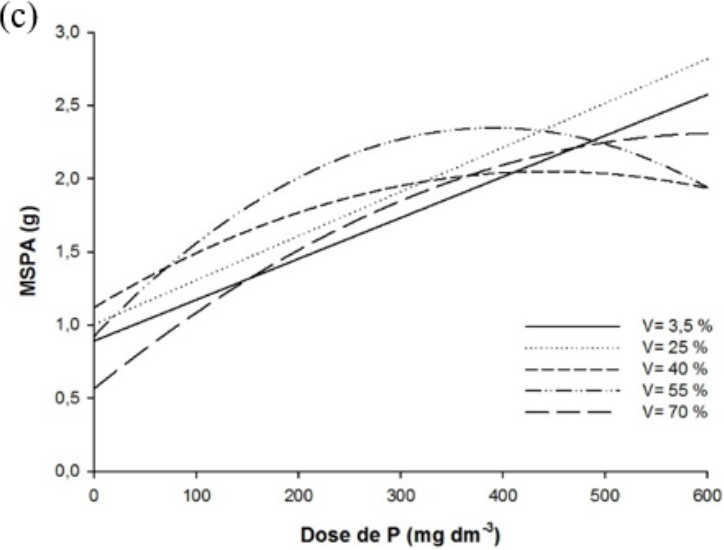

(e)

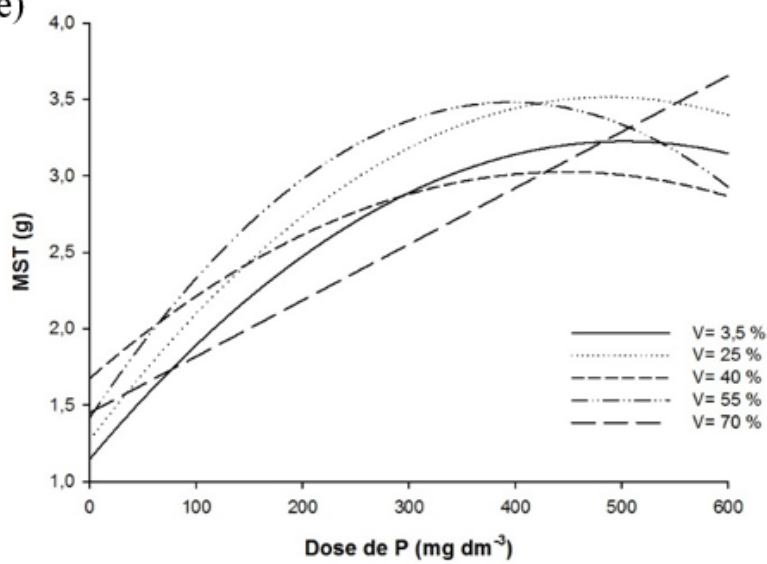

(b)

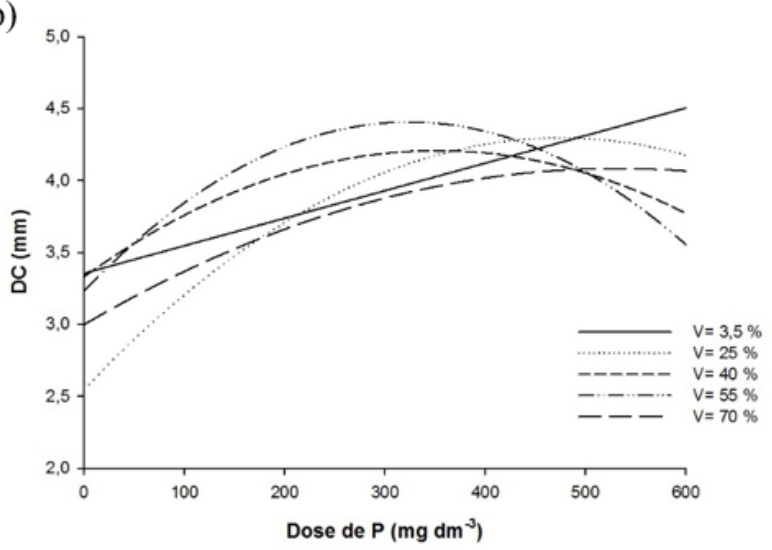

(d)

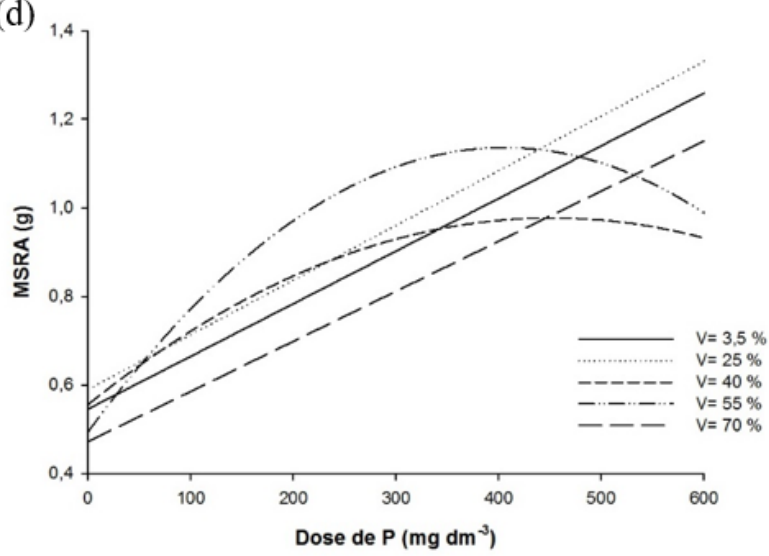

(f)

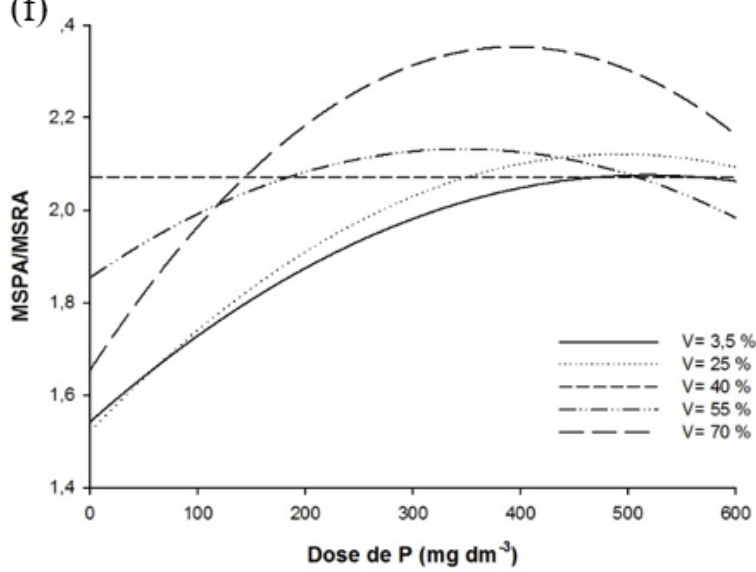

FIGURA 1: Altura da parte aérea (H) (a), diâmetro do coleto (DC) (b), massa de matéria seca da parte aérea (MSPA) (c), massa de matéria seca da raiz (MSRA) (d), massa de matéria seca total (MST) (e), relação entre massa de matéria seca da parte aérea e massa de matéria seca da raiz (MSPA/MSRA) (f), para mudas de Cassia grandis aos 65 dias após a repicagem, em resposta à adubação fosfatada em diferentes saturações por base do substrato.

FIGURE 1: Height of the aerial part (H) (a), collar diameter (DC) (b), dry matter of shoot (MSPA) (c), dry matter of root (MSRA) (d), total dry matter (MST) (e), relation between dry matter of shoot and dry matter of root (MSPA/MSRA) (f), for Cassia grandis seedlings in response to phosphate fertilization in different substratum base saturation. The morphologic characteristics were evaluated at 65 days after transplanting. 
TABELA 3: Equações de regressão para a altura da parte aérea (H), diâmetro do coleto (DC), massa de matéria seca da parte aérea (MSPA), massa de matéria seca da raiz (MSRA), massa de matéria seca total (MST), relação entre massa de matéria seca da parte aérea e massa de matéria seca da raiz (MSPA/MSRA), e índice de qualidade de Dickson (IQD) como variáveis dependentes das doses de $\mathrm{P}$ nas diferentes saturações por bases do substrato, para produção de mudas de Cassia grandis aos 65 dias após a repicagem.

TABLE 3: Regression equations for height of the aerial part (H), collar diameter (DC), dry matter of shoot (MSPA), dry matter of root (MSRA), total dry matter (MST), relation between dry matter of shoot and dry matter of root (MSPA/MSRA), and Dickson quality index (IQD) as dependent variables of $\mathrm{P}$ doses in different substratum base saturation for Cassia grandis seedling production at 65 days after transplanting.

\begin{tabular}{|c|c|c|c|c|}
\hline Variável dependente & $\mathrm{V}(\%)$ & Equação & $\mathrm{R}^{2}$ & p-valor \\
\hline \multirow{5}{*}{$\mathrm{H}(\mathrm{cm})$} & 3,5 & $\hat{\mathrm{Y}}=16,4039+0,04131 * * \mathrm{P}-0,00004888^{*} \mathrm{P}^{2}$ & 47 & 0,0013 \\
\hline & 25 & $\hat{\mathrm{Y}}=13,8301+0,06471 * \mathrm{P}-0,00007840 * \mathrm{P}^{2}$ & 63 & 0,0000 \\
\hline & 40 & $\hat{\mathrm{Y}}=19,6466+0,02385^{\circ} \mathrm{P}-0,00002847^{0,1602} \mathrm{P}^{2}$ & 22 & 0,0710 \\
\hline & 55 & $\hat{\mathrm{Y}}=17,7921+0,05987 * * \mathrm{P}-0,00009355^{*} * \mathrm{P}^{2}$ & 52 & 0,0004 \\
\hline & 70 & $\hat{\mathrm{Y}}=16,2255+0,04123^{* *} \mathrm{P}-0,00003930^{\circ} \mathrm{P}^{2}$ & 56 & 0,0002 \\
\hline \multirow{5}{*}{$\mathrm{DC}(\mathrm{mm})$} & 3,5 & $\hat{\mathrm{Y}}=3,3548+0,001913 * * \mathrm{P}$ & 27 & 0,0087 \\
\hline & 25 & $\hat{\mathrm{Y}}=2,5473+0,007342 * * \mathrm{P}-0,000007712 * \mathrm{P}^{2}$ & 52 & 0,0004 \\
\hline & 40 & $\hat{\mathrm{Y}}=3,3314+0,004979 * \mathrm{P}-0,000007077 * \mathrm{P}^{2}$ & 24 & 0,0543 \\
\hline & 55 & $\hat{\mathrm{Y}}=3,2321+0,007243^{* *} \mathrm{P}-0,00001118^{*} * \mathrm{P}^{2}$ & 35 & 0,0114 \\
\hline & 70 & $\hat{\mathrm{Y}}=2,9983+0,004079 * \mathrm{P}-0,000003835^{0,1311} \mathrm{P}^{2}$ & 48 & 0,0010 \\
\hline \multirow{5}{*}{$\operatorname{MSPA}(\mathrm{g})$} & 3,5 & $\hat{\mathrm{Y}}=0,8919+0,002803 * * \mathrm{P}$ & 44 & 0,0004 \\
\hline & 25 & $\hat{\mathrm{Y}}=1,003994+0,003022 * * \mathrm{P}$ & 50 & 0,0002 \\
\hline & 40 & $\hat{\mathrm{Y}}=1,1201+0,004181^{\circ} \mathrm{P}-0,000004706^{0,1724} \mathrm{P}^{2}$ & 26 & 0,0421 \\
\hline & 55 & $\hat{\mathrm{Y}}=0,9237+0,007278^{*} * \mathrm{P}-0,000009309 * * \mathrm{P}^{2}$ & 48 & 0,0015 \\
\hline & 70 & $\hat{\mathrm{Y}}=0,5662+0,005642 * \mathrm{P}-0,000004562^{0,1865} \mathrm{P}^{2}$ & 56 & 0,0002 \\
\hline \multirow{5}{*}{$\operatorname{MSRA}(g)$} & 3,5 & $\hat{\mathrm{Y}}=0,5457+0,001188^{*} * \mathrm{P}$ & 32 & 0,0039 \\
\hline & 25 & $\hat{\mathrm{Y}}=0,5897+0,001234 * * \mathrm{P}$ & 42 & 0,0008 \\
\hline & 40 & $\hat{\mathrm{Y}}=0,5558+0,001863^{\circ} \mathrm{P}-0,000002061^{0,1725} \mathrm{P}^{2}$ & 28 & 0,0339 \\
\hline & 55 & $\hat{\mathrm{Y}}=0,4937+0,003166 * * \mathrm{P}-0,000003903 * \mathrm{P}^{2}$ & 39 & 0,0072 \\
\hline & 70 & $\hat{\mathrm{Y}}=0,4724+0,001130 * * \mathrm{P}$ & 35 & 0,0023 \\
\hline \multirow{5}{*}{ MST (g) } & 3,5 & $\hat{\mathrm{Y}}=1,1480+0,008275^{*} \mathrm{P}-0,000008242^{0,1663} \mathrm{P}^{2}$ & 41 & 0,0052 \\
\hline & 25 & $\hat{\mathrm{Y}}=1,2723+0,009191 * \mathrm{P}-0,000009419^{\circ} \mathrm{P}^{2}$ & 51 & 0,0012 \\
\hline & 40 & $\hat{\mathrm{Y}}=1,6759+0,006043^{\circ} \mathrm{P}-0,000006767^{0,1678} \mathrm{P}^{2}$ & 27 & 0,0371 \\
\hline & 55 & $\hat{\mathrm{Y}}=1,4175+0,01044 * * \mathrm{P}-0,00001321 * \mathrm{P}^{2}$ & 46 & 0,0023 \\
\hline & 70 & $\hat{\mathrm{Y}}=1,4512+0,003672 * * \mathrm{P}$ & 37 & 0,0016 \\
\hline \multirow{5}{*}{ MSPA/MSRA } & 3,5 & $\hat{\mathrm{Y}}=1,5424+0,002058 * \mathrm{P}-0,000001986^{0,1347} \mathrm{P}^{2}$ & 45 & 0,0018 \\
\hline & 25 & $\hat{\mathrm{Y}}=1,5214+0,002435^{* *} \mathrm{P}-0,000002473^{\circ} \mathrm{P}^{2}$ & 51 & 0,0005 \\
\hline & 40 & $\hat{\mathrm{Y}}=\overline{\mathrm{Y}}$ & & \\
\hline & 55 & $\hat{\mathrm{Y}}=1,8542+0,001603^{\circ} \mathrm{P}-0,000002314^{0,1339} \mathrm{P}^{2}$ & 13 & 0,2348 \\
\hline & 70 & $\hat{\mathrm{Y}}=1,6542+0,003543^{\circ} \mathrm{P}-0,000004492^{0,1156} \mathrm{P}^{2}$ & 22 & 0,0768 \\
\hline \multirow{5}{*}{ IQD } & 3,5 & $\hat{\mathrm{Y}}=0,1947+0,0005001 * * \mathrm{P}$ & 39 & 0,0012 \\
\hline & 25 & $\hat{\mathrm{Y}}=0,2251+0,0004797 * \mathrm{P}$ & 36 & 0,0019 \\
\hline & 40 & $\hat{\mathrm{Y}}=0,2069+0,0008410^{*} \mathrm{P}-0,000001034^{\circ} \mathrm{P}^{2}$ & 27 & 0,0347 \\
\hline & 55 & $\hat{\mathrm{Y}}=0,2128+0,001103^{*} \mathrm{P}-0,000001378^{\circ} \mathrm{P}^{2}$ & 31 & 0,0204 \\
\hline & 70 & $\hat{\mathrm{Y}}=0,1843+0,0003675^{* * \mathrm{P}}$ & 38 & 0,0014 \\
\hline
\end{tabular}

Em que: Coeficiente significativo a $1 \%(* *), 5 \%(*)$ e $10 \%\left({ }^{\circ}\right)$ de probabilidade. 
sido considerada uma das melhores para predizer a qualidade de mudas, porém apresenta o inconveniente de sua determinação não ser viável em muitos viveiros, principalmente por ser destrutiva e necessitar de estufas (GOMES; PAIVA, 2012).

As características morfológicas podem ser consideradas isoladamente, todavia, recomenda-se que os seus valores sejam relacionados. A distribuição de massa na muda é indicada pela relação MSPA/MSRA. Segundo Gomes e Paiva (2012), a melhor relação entre a massa de matéria seca da parte aérea e a respectiva massa de matéria seca de raiz é equivalente a 2,0 para mudas de qualidade. A dose de P que proporcionou esse resultado foi de $323 \mathrm{mg} \mathrm{dm}^{-3}$ na saturação por bases natural do solo, $271 \mathrm{mg} \mathrm{dm}^{-3}$ para a de $25 \%, 108$ $\mathrm{mg} \mathrm{dm} \mathrm{d}^{-3}$ para a de $55 \%$ e $114 \mathrm{mg} \mathrm{dm}^{-3}$ para a de $70 \%$.

$\mathrm{O}$ índice de qualidade de Dickson pondera os resultados de várias características importantes empregadas para a avaliação da qualidade, e é considerado como um dos melhores indicadores da qualidade das mudas, pois no seu cálculo são considerados a robustez e o equilíbrio da distribuição de massa na muda (FONSECA et al., 2002). Quanto maior for o valor desse índice, melhor será o padrão de qualidade das mudas (GOMES; PAIVA, 2012). Para a saturação por bases natural do solo, de $25 \%$ e $70 \%$ observa-se resposta linear do IQD em relação às doses de fósforo testadas (Figura 2). O maior IQD $(0,51)$, na saturação por bases de $25 \%$, foi obtido na dose de $600 \mathrm{mg} \mathrm{dm}^{-3}$ de $\mathrm{P}$.

Foram constatadas correlações positivas e significativas entre as variáveis estudadas (Tabela 4). A correlação, acima de 0,75 , entre as variáveis dependentes indica que a observação de uma variável possibilitaria inferir sobre as outras. Nesse sentido, a recomendação da adubação e da correção do solo para obter o maior IQD é justificável uma vez que esse índice se correlaciona com as características avaliadas. Ressalta-se, também, que a observação da altura das mudas no viveiro é um método para inferir a respeito da qualidade das mesmas, uma vez que essa característica apresentou correlação com as outras variáveis, além de ser viável operacionalmente, uma vez que não se trata de um método destrutivo.

Assim como nesse estudo, respostas positivas à adubação fosfatada têm sido registradas por diversos autores para espécies arbóreas nativas, como freijó (Cordia goeldiana) (FERNANDES et al., 2007); angico-vermelho (Parapiptadenia rigida) (SCHUMACHER; CECONI; SANTANA, 2004; GONÇALVES et al., 2008); garapa (Apuleia leiocarpa) (GOMES et al., 2008); angico-branco (Anadenanthera colubrina) (GOMES et al., 2004), fedegoso (Senna macranthera) (CRUZ et al., 2011), açoita-cavalo (Luehea divaricata) (CECONI et al., 2006), sansão-do-campo (Mimosa caesalpiniaefolia) (GONÇALVES et al., 2010), erva-mate (Ilex paraguariensis) (SANTIN et al., 2008), aroeira (Lithraea molleoides), aroeirinha

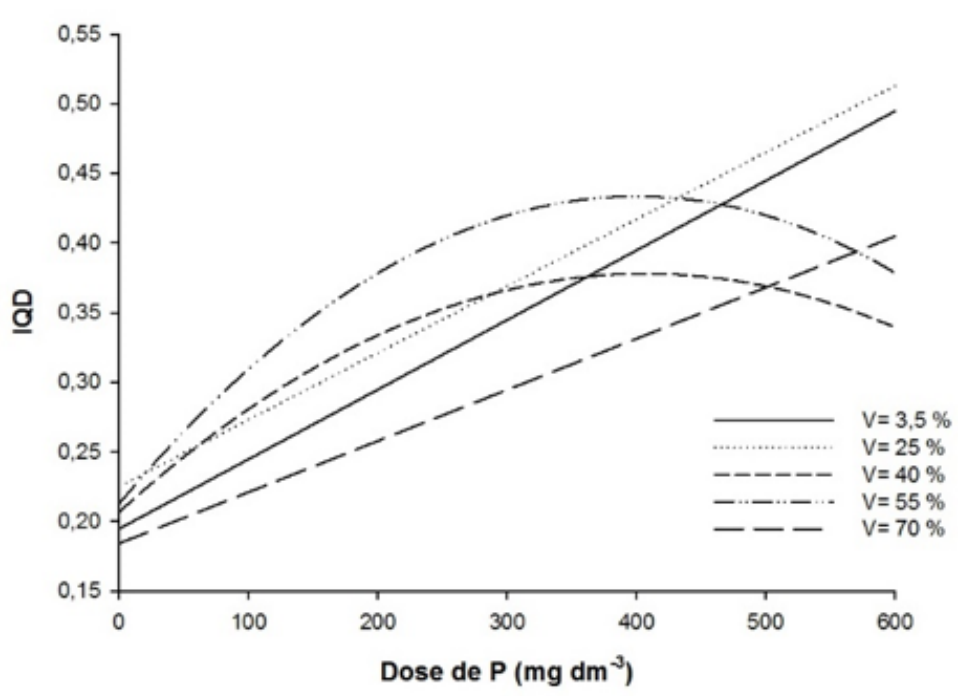

FIGURA2: Índice de qualidade de Dickson (IQD) de mudas de Cassia grandis, aos 65 dias após a repicagem, em resposta à adubação fosfatada nas diferentes saturações por base do substrato.

FIGURE 2: Dickson's quality index for Cassia grandis seedlings in response to phosphate fertilization in different substratum base saturation. It was evaluated at 65 days after transplanting. 
TABELA 4: Coeficiente de correlação de Pearson entre as variáveis estudadas: altura da parte aérea $(\mathrm{H})$, diâmetro do coleto (DC), massa de matéria seca da parte aérea (MSPA), massa de matéria seca da raiz (MSRA), massa de matéria seca total (MST), e índice de qualidade de Dickson (IQD).

TABLE 4: Pearson's correlation coefficient between variables: height of the aerial part $(\mathrm{H})$, collar diameter (DC), dry aerial part matter (MSPA), dry root matter (MSRA), dry total matter (MST) and Dickson's quality index (IQD).

\begin{tabular}{lccccc}
\hline \multicolumn{1}{c}{ Características } & DC $(\mathrm{mm})$ & MSPA $(\mathrm{g})$ & MSRA $(\mathrm{g})$ & MST $(\mathrm{g})$ & IQD \\
\hline H $(\mathrm{cm})$ & $0,81^{* *}$ & $0,90^{* *}$ & $0,83^{* *}$ & $0,89^{* *}$ & $0,81^{* *}$ \\
DC $(\mathrm{mm})$ & & $0,79^{* *}$ & $0,76^{* *}$ & $0,79^{* *}$ & $0,85^{* *}$ \\
MSPA $(\mathrm{g})$ & & $0,95^{* *}$ & $0,99^{* *}$ & $0,95^{* *}$ \\
MSRA $(\mathrm{g})$ & & & $0,98^{* *}$ & $0,97^{* *}$ \\
MST $(\mathrm{g})$ & & & & $0,97^{* *}$ \\
\hline
\end{tabular}

Em que: ** significativo a $1 \%$ de probabilidade.

(Schinus terebinthifolius), sesbânia (Sesbania virgata) (SANTOS et al., 2008), angico-amarelo (Peltophorum dubium) (SCHUMACHER; CECONI; SANTANA, 2003), sendo a concentração ótima variável com a espécie e tipo de substrato utilizado.

\section{CONCLUSÕES}

A adubação fosfatada influencia positivamente o crescimento e a qualidade das mudas de cássiarosa, nas condições estudadas. A dose de fósforo adequada para a produção de mudas de cássia-rosa foi de $600 \mathrm{mg} \mathrm{dm}^{-3}$ na saturação por bases de $25 \%$.

\section{AGRADECIMENTOS}

À Coordenação de Aperfeiçoamento de Pessoal de Nível Superior (CAPES), pela concessão da bolsa de mestrado e ao CNPq pela concessão da bolsa de produtividade em pesquisa.

\section{REFERÊNCIAS}

ALVAREZ, V. H. V. et al. Poda de raízes e adubação para crescimento do cafeeiro cultivado em colunas de solo. Revista Brasileira de Ciência do Solo, Viçosa, MG, v. 30, n. 1, p. 111-119, 2006.

ALVAREZ, V. H. V.; RIBEIRO, A. C. Calagem. In: RIBEIRO, A. C.; GUIMARÃES, P. T. G.; ALVAREZ, V. H. V. (Ed.). Recomendações para o uso de corretivos e fertilizantes em Minas Gerais - $5^{\text {a }}$ Aproximação. Viçosa: Comissão de Fertilidade do Solo do Estado de Minas Gerais, 1999. p. 43-61.

BALIEIRO, F. C. et al. Formação de mudas de Acacia holosericea e Acacia auriculiformis: resposta à calagem, fósforo, potássio e enxofre. Revista Árvore, Viçosa, MG, v. 25, n. 2, p. 183-191, 2001.

BERNARDINO, D. C. S. et al. Crescimento e qualidade de mudas de Anadenanthera macrocarpa (Benth.) Brenan em resposta à saturação por bases do substrato. Revista Árvore, Viçosa, MG, v. 29, n. 6, p. 863-870, 2005.

CAIONE, G.; LANGE, A.; SCHONINGER, E. L. Crescimento de mudas de Schizolobium amazonicum (Huber ex Ducke) em substrato fertilizado com nitrogênio, fósforo e potássio. Scientia Forestalis, Piracicaba, v. 40, n. 94, p. 213-221, 2012.

CALDEIRA JÚNIOR, C. F. et al. Níveis de saturação por bases para a produção de mudas de nim-indiano (Azadirachta indica A. Juss). Revista Brasileira de Plantas Medicinais, Botucatu, v. 9, n. 4, p. 80-85, 2007.

CARVALHO, P. E. R. Cássia-Rósea. Colombo: Embrapa Florestas, 2006. 8 p. (Circular Técnica, 117). 
CARVALHO, P. E. R. Espécies florestais brasileiras: recomendações silviculturais, potencialidades e uso da madeira. Brasília: EMBRAPA - CNPF/SPI, 1994. 640 p.

CECONI, D. E. et al. Crescimento de mudas de açoita-cavalo (Luehea divaricata Mart.) sob influência da adubação fosfatada. Cerne, Lavras, v. 12, n. 3, p. 292-299, 2006.

CRUZ, C. A. F. et al. Crescimento e qualidade de mudas de Fedegoso cultivadas em latossolo vermelhoamarelo em resposta a macronutrientes. Scientia Forestalis, Piracicaba, v. 39, n. 89, p. 21-33, 2011.

CRUZ, C. A. F. et al. Efeito de diferentes níveis de saturação por bases no desenvolvimento e qualidade de mudas de ipê-roxo (Tabebuia impetiginosa (Mart.) Standley). Scientia Forestalis, Piracicaba, n. 66, p. 100-107, 2004.

DONAGEMA, G. K. Manual de métodos de análise de solos. Rio de Janeiro: Embrapa, 2011. 230 p.

EMBRAPA. Centro Nacional de Pesquisa de Solos. Sistema brasileiro de classificação de solos. 3. ed. Brasília: Embrapa, 2013. 353 p.

FERNANDES, A. R. et al. Crescimento e absorção de nutrientes por mudas de freijó (Cordia goeldiana Huber) em função de doses de fósforo e de zinco. Revista Árvore, Viçosa, MG, v. 31, n. 4, p. 599-608, 2007.

FONSECA, E. P. et al. Padrão de qualidade de mudas de Trema micrantha (L.) Blume, produzidas sob diferentes períodos de sombreamento. Revista Árvore, Viçosa, MG, v. 26, n. 4, p. 515-523, 2002.

FURTINI NETO, A. E. et al. Acidez do solo, crescimento e nutrição mineral de algumas espécies arbóreas, na fase de muda. Cerne, Lavras, v. 5, n. 2, p. 1-12, 1999.

FURTINI NETO, A. E. et al. Fertilização em reflorestamentos com espécies nativas. In: GONÇALVES, J. L. M.; BENEDETI, V. (Ed.). Nutrição e fertilização florestal. Piracicaba: IPEF, 2005. p. 351-384.

GOMES, J. M. et al. Parâmetros morfológicos na avaliação da qualidade de mudas de Eucalyptus grandis. Revista Árvore, Viçosa, MG, v. 26, n. 6, p. 655-664, 2002.

GOMES, J. M.; PAIVA, H. N. Viveiros florestais: propagação sexuada. Viçosa: Editora UFV, 2012. 116 p. GOMES, K. C. O. et al. Influência da saturação por bases e do fósforo no crescimento de mudas de angicobranco. Revista Árvore, Viçosa, MG, v. 28, n. 6, p. 785-792, 2004.

GOMES, K. C. O. et al. Crescimento de mudas de garapa em resposta à calagem e ao fósforo. Revista Árvore, Viçosa, MG, v. 32, n. 3, p. 387-394, 2008.

GONÇALVES, E. O. et al. Crescimento de mudas de angico-vermelho (Anadenanthera macrocarpa (Benth.) Brenan) sob diferentes doses de macronutrientes. Revista Árvore, Viçosa, MG, v. 32, n. 6, p. 1029-1040, 2008.

GONÇALVES, E. O. et al. Crescimento de mudas de sansão-do-campo (Mimosa caesalpiniaefolia Benth.) sob diferentes doses de macronutrientes. Scientia Forestalis, Piracicaba, v. 38, n. 88, p. 599-609, 2010.

GONÇALVES, J. L. M. et al. Produção de mudas de espécies nativas: substrato, nutrição, sombreamento e fertilização. In: GONÇALVES, J. L. M.; BENEDETI, V. (Ed.). Nutrição e fertilização florestal. Piracicaba: IPEF, 2005. p. 309-350.

INSTITUTO NACIONAL DE METEOROLOGIA (Brasil). Normais climatológicas 1961-1990. Brasília: SPI/EMBRAPA, 1992.

LORENZI, H. Árvores brasileiras: manual de identificação e cultivo de plantas arbóreas nativas do Brasil. 4. ed. Nova Odessa: Instituto Plantarum, 2002. v. 1.

MARSCHNER, H. Mineral nutrition of higher plants. 2. ed. San Diego: Academic Press, 1995. 888 p. NOVAIS, R. F. et al. Fósforo. In: NOVAIS, R. F. et al. Fertilidade do solo. Viçosa: Sociedade Brasileira de Ciência do Solo, 2007. p. 472-537.

PARROTTA, J.A. The role of plantation forests in rehabilitating degraded tropical ecosystems. Agriculture, Ecosystems and Environment, Amsterdam, v. 41, n. 2, p. 115-133, 1992.

PASSOS, M. A. A. Efeito da calagem e de fósforo no crescimento inicial da algaroba (Prosopis juliflora (SW) DC). 1994. 57 f. Tese (Doutorado em Fitotecnia) - Universidade Federal de Viçosa, Viçosa, 1994.

SANTIN, D. et al. Crescimento de mudas de erva-mate fertilizadas com N, P e K. Scientia Agraria, Curitiba, v. 9, n. 1, p. 59-66, 2008.

SANTOS, J. Z. L. et al. Crescimento, acúmulo de fósforo e frações fosfatadas em mudas de sete espécies arbóreas nativas. Revista Árvore, Viçosa, MG, v. 32, n. 5, p. 799-807, 2008.

SCHUMACHER, M. V.; CECONI, D. E.; SANTANA, C. A. Influência de diferentes doses de fósforo 
no crescimento de plantas de Peltophorum dubium (Sprengel) Taubert. Boletim de Pesquisa Florestal, Colombo, n. 47, p. 99-114, 2003.

SCHUMACHER, M. V.; CECONI, D. E.; SANTANA, C. A. Influência de diferentes doses de fósforo no crescimento de mudas de angico-vermelho (Parapiptadenia rigida (Bentham) Brenan). Revista Árvore, Viçosa, MG, v. 28, n. 1, p. 149-155, 2004.

SILVA, A. H.; PEREIRA, J. S.; RODRIGUES, S. C. Desenvolvimento inicial de espécies exóticas e nativas e necessidade de calagem em área degradada do Cerrado no triângulo mineiro (Minas Gerais, Brasil). Agronomía Colombiana, Bogotá, v. 29, n. 2, p. 287-292, 2011.

SOUZA, C. A. M. et al. Crescimento em campo de espécies florestais em diferentes condições de adubações. Ciência Florestal, Santa Maria, v. 16, n. 3, p. 243-249, 2006.

SOUZA, P. H. et al. Crescimento e qualidade de mudas de Senna macranthera (Collad.) Irwin et Barn. em resposta à calagem. Revista Árvore, Viçosa, MG, v. 34, n. 2, p. 233-240, 2010.

SOUZA, P. H. et al. Influência da saturação por bases do substrato no crescimento e qualidade de mudas de Machaerium nictitans (Vell.) Benth. Revista Árvore, Viçosa, MG, v. 32, n. 2, p. 193-201, 2008.

VALE, F. R. et al. Crescimento radicular de espécies florestais em solo ácido. Pesquisa Agropecuária Brasileira, Brasília, v. 31, n. 9, p. 609-616, 1996.

VAN RAIJ, B.; SANCCHETTO, M. T. D. Correlações entre o pH e o grau de saturação em bases nos solos com horizonte B textural e horizonte B latossólico. Bragantia, Campinas, v. 27, n. 17, p. 193-200, 1968. 